\title{
Espace et immensité intime dans La nuit de feu d'Éric Emmanuel Schmitt
}

\section{Space and Intimate Immensity in Éric Emmanuel Schmitt's Night of fire}

\author{
Hafid ABOUELKACEM ${ }^{1}$ [D
}

'Doctorant, Université lbno Zohr, Faculté des Lettres et des Sciences Humaines, Département de Langue et de littérature francaises, Agadir-Maroc

ORCID: H.A. 0000-0003-4391-8658

\author{
Corresponding author: \\ Hafid ABOUELKACEM, \\ Université lbno Zohr, Faculté des Lettres \\ et des Sciences Humaines, Départemen \\ de Langue et de littérature francaises, \\ Agadir-Maroc
}

E-mail: haafidabouelkacem@gmail.com

Submitted: 27.02 .2020

Revision Requested: 28.08.2020

Last Revision Received: 01.09.2020

Accepted: 26.10 .2020

Citation: Abouelkacem, H. (2020). Espace et immensité intime dans La nuit de feu d'Éric Emmanuel Schmitt. Litera, 30(2), 505-520

https://doi.org/10.26650/LITERA2020-0042

\section{RÉSUMÉ}

Notre étude vise l'« immense » dans La Nuit de feu en ce qu'il permet de mettre en lumière le désert. En cela que nous avons recouru à la notion de l'immensité intime théorisée dans la philosophie de Gaston Bachelard. É. E. Schmitt s'est emparé du « je » que d'ailleurs il n'aime pas pour dire quelle révélation il avait reçu et quel homme il est devenu après l'appel intime de l'immense. Partant du fait que l'immensité du désert retentit en immensité intime, nous avons montré à quel point le désert est un espace de renaissance et de révélation. Le texte est ainsi jonché des marques de l'immensité, cela est particulièrement dû à la rêverie de l'immense qui s'est activée dans l'intimité du narrateur. Sa contemplation de l'infini a fait que son corps a subit une dilatation, ou encore une nette expansion. Ce qui corrobore le fait que le regard absorbe l'immensité du désert. Le dédoublement du corps est aussi nettement décelable chez le narrateur. Deux êtres logent en lui. Le phénomène d'expansion se produit aussi sous le mode d'une ascension. La dilatation du corps à laquelle la rêverie a donné naissance permet au narrateur de se libérer de la pesanteur afin de respirer cosmiquement jusqu'à se trouver dans un ailleurs intime, spatial et atemporel.

Mots-clés: Espace, immensité intime, désert, imagination, rêverie

\section{ABSTRACT}

Our study aims at the "immense" in The Night of fire that brings the desert to light. Furthermore, we have resorted to the notion of intimate immensity theorized in Gaston Bachelard's philosophy. After this intimate call of the immense, É. E. Schmitt seized the "l" that he does not like to say what revelation he had received and what man he has become. We have shown to what extent the desert is a space of rebirth and revelation, starting from the fact that the immensity of the desert resounds in intimate vastness. The text is thus strewn with the marks of immensity; this is particularly due to the reverie of the immense that has been activated in the intimacy of the narrator. His contemplation of the infinite has caused his body to undergo a clear expansion or even a dilation. This corroborates the fact that the gaze absorbs the immensity of the desert. The doubling of the body is also clearly detectable in the narrator. Two beings lodge in him. The phenomenon of expansion also occurs in the form of an ascension. To breathe cosmically until he finds himself in an intimate, spatial, and timeless elsewhere, the dilation of the body to which reverie has given birth allows the narrator to free himself from gravity.

Keywords: Space, intimate immensity, desert, imagination, reverie 


\section{EXTENDED ABSTRACT}

Man is only the mirror of immensity according to Bachelard. It is inside man that space spreads out. Because being this passage leads to the reverie of intimate immensity, the look is of major importance. You could say that the eye contemplates the infinity of space and reflects the contemplated landscape. By contemplation and by the look, the intimate immensity is enlarged. It must be said that until they find themselves in a phenomenon of fusion, it is through greatness that intimate space and the space of the world become consonant.

According to Bachelard, daydreaming is a bit peculiar for those who venture into the desert in that the immensity lived in the desert by a dreamer sound in an intensity of being intimate. You can see in the Schmittian desert the space of a rebirth. It is also the space for a fresh start. All of this is experienced intimately by the narrator. It seems opportune to proceed by taking samples from several passages in the novel with the intention of shedding light on this lived intimacy.

The first notch that we opened was that of the recurrence of the marks of immensity to cross the Schmittian text as they are felt by the characters. Wherever there are characters contemplating the infinity of the desert, it seems that these brands dot the whole fabric of the novel in several ways. The novel is riddled with these marks of vastness, sometimes in explicit form and sometimes allusive. Words are varied proofs: To grasp their meaning is to understand the reveries that open, in the soul of the narrator, under the sign of the words "infinite" and "immense."Indeed, these words mark in a natural way the intimate immensity because of having intimate resonances.

Regarding humans who inhabit it or cross it, it is quite clear that the figure of the desert in the text is also constructed. Let us also add that there are several references to the human body, to its movements, and to its sensations. In this perspective, it seemed important to us to evoke the posture of the body of the narrator. É. E. Schmitt, lost in the desert for one night, he will be "lying on his back, in a lying position, the figure facing the evening star" (Schmitt, 2015, p. 103) the motionless body facing the sky. This suggests that this posture is only a preamble to a movement that will be accomplished in stillness otherwise, a completely internal movement: "I rise, I exceed the sand, the mass of rocks, and ... I float" (Schmitt, 2015, p. 106). This movement is unquestionably imaginary as it describes the epiphany lived by the narrator in the 
desert. We must see in this movement of the immobile being an attempt to transcend time and space.

The immense lodge peacefully in the reverie of the narrator. The vastness of the outside is transformed into the vastness of the interior. It is all thanks to the soul that meditates and dreams that tries to find in an object the nest of immensity and to the "contemplation of grandiose spectacles".

Two bodies are put in parallel that one can grasp as a detachment, which occurs fortuitously in the total silence and the heat of the sands: one freed, free by a force that maintains it above and the other below prisoner huge sands that block it. If you think otherwise. It is indeed two spaces: one is intimate, and the other is exterior. The intimate is freed by the force of the immense, and the exterior space is that of the prisoner body, surrounded by the immense dunes. However, it is only when the narrator has become motionless, blocked by the heat of the sand, that his true inner journey begins.

The narrator launches into the reverie of the immense reaching an exterior and intimate elsewhere, spatial and temporal. Indeed, the experience of intimate immensity confuses not only the borders between the body and space but also it makes the borders between space and time porous, which gives birth to an "elsewhere." In the sense of a zone between the real and the imaginary. 


\section{Introduction}

La Nuit de feu est le roman de l'« immense » en ce qu'il met en lumière le désert, ce vaste étendu aux extrêmes conditions. Le désert y est présenté comme un espace infini, immense conduisant tout aventurier à renaitre et à se purifier. Inspiré par ses immenses dunes et ses courbes éblouissantes, É. E. Schmitt se voit naitre deux fois « une fois à Lyon en 1960, une fois au Sahara en $1989 »$ (2015, p. 141). C'est ainsi qu'il retrousse ses manches pour nous raconter le souvenir d'une nuit dans le « sarcophage de sable » gravé dans sa mémoire, récit d'une épiphanie vécu sous le soleil intense du désert de Tamanrasset. C'est une nuit mystique, enfoui dans le lit de sable contemplant l'infini céleste, une nuit « irradiée » qui mène à la métamorphose de l'être. Il reçoit une révélation au contact de la force qui régit le désert. C'est ainsi qu'on ne sort pas indemne du désert narré par l'auteur. La raison du philosophe se brise devant celle du désert grâce à la force de l'infini terrestre et céleste, force qui sait faire sentier vers l'espace intime de tout contemplateur. L'écrivain français s'empare du «je » que d'ailleurs il n'aime pas pour dire quelle révélation il avait reçu et quel homme il est devenu après cet appel intime de l'immense. Partant du fait que l'immensité du désert retentit en immensité intime, nous allons montrer dans quelle mesure la notion bachelardienne de l'immensité intime enrichit et éclaire la lecture de La nuit de feu. Au commencement, un survol théorique axé sur l'étude de l'espace en littérature nous est nécessaire. Une précision théorique du concept de l'espace tel que pensé par les phénoménologues serait aussi indispensable. Il s'agira en suite de présenter la méthode bachelardienne d'analyse de l'espace romanesque. C'est ainsi que nous allons expliciter la notion d'immensité intime. Il est de circonstance de se demander comment É. E. Schmitt déploie à travers son roman aux allures d'autobiographie et de conte philosophique, les marques de l'immensité et du corps. Nous allons montrer aussi les manifestations d'expansion corporelle et d'ascension qui consacrent l'immensité vécu par l'auteur. Ensuite, nous mettrons en évidence le phénomène de fusion entre le corps et l'espace. Enfin, on verra également comment l'immensité donne accès à un « ailleurs » à la fois extérieur et intime.

\section{Sens phénoménologique de l'espace}

L'héritage kantien a fait que l'espace n'est plus le milieu dans lequel les choses sont disposées, mais bien le moyen par lequel la position des choses devient possible (Merleau-Ponty, 2005, p. 290). Merleau-Ponty s'inscrit dans l'esprit des recherches

d'Edmund Husserl, il voit dans l'espace l'expression du corps lui-même car il constitue 
le moyen concret par lequel l'être s'insère dans le monde phénoménal (Merleau-Ponty, 2005). Sa conception peut donc être saisie comme le rapport entre le corps et l'univers. De là, à dire qu'à l'origine de la spatialité, on trouve le corps, le sujet corporel. Les clefs de l'analyse de l'espace résultent de ce que Bertrand Levy nomme « la corporéité de l'être » (Levy, 1987, p. 127). Ce qui montre en corolaire que l'espace n'est pas antérieur à toute expérience mais il en tire son origine. Bachelard met en lumière la notion de $\mathrm{I}^{\prime}$ « habiter » (Bachelard, 1957) notion traduisant la volonté de l'homme d'aménager un espace réel ou imaginaire qui lui est propre. Son approche concède des éléments concrets pour l'analyse de l'espace envisagé dans sa dimension domestique et cosmique. Le théoricien dévoile l'enracinement de l'homme dans un coin du monde. Il voit que notre présence dans un coin du monde fait qu'il dit beaucoup sur la relation que nous entretenons avec soi et avec la vie. Habiter peut être compris comme geste d'ouverture au monde par la création d'un espace unique. Sa conception s'inscrit dans la continuité de l'apport majeurs de Martin Heidegger à savoir que l'« homme habite en poète » (Heidegger, 1988, p. 224) parce qu'il investit l'espace dans lequel il s'agite par ses mouvements, par ses sens et son corps. Dans la poétique de l'espace, Gaston Bachelard propose de cerner la signification du verbe Habiter à travers ce qu'il appelle la « topoanalyse » de l'espace vécu. Sa pensée se résume par le fait que certaines parties de la maison se transforment par le biais de l'imagination en image poétique de l'espace. II met également en lumière l'idée de l'espace intime. Dans le même ordre d'idées, Bachelard considère que l'homme imagine le monde, cela lui accorde « un chez soi en expansion » autrement dit, «l'envers du chez soi de la chambre » (Bachelard, 1957, p. 152). Ce qui fait de l'espace un pur produit d'activités matérielles et intellectuelles. En ce sens, il se voit clair que l'espace résulte de l'expérience vécu de l'homme et de ce fait, il devient l'expression du corps humain.

\section{Espace et littérature}

Bien avant le tournant spatial dans les sciences sociales et humaines (Soja, 2009), la théorie littéraire a connu d'importantes études sur l'espace. On peut citer l'apport majeur de sémioticiens à l'instar de Algirdas Julien Greimas pour qui, le langage spatial est particulièrement intéressant en ce qu'il a la capacité de " pouvoir s'ériger en un métalangage capable de parler de tout autre chose que de l'espace et d'être un langage par lequel la société se signifie elle-même » (Alonso Aldama, 2009, citant Greimas 1976, p. 130-131). Denis Bertrand propose pour sa part une sémiotique concrète qui se donne pour tâche d'explorer la topologie du roman sans se fier à la thématique 
substantialiste et ses pièges ou à l'illusion référentielle. Pour ce faire, une attention de principe, selon lui, est nécessaire " aux relations qui unissent les configurations spatiales entre elles et à l'ensemble des autres composantes : notamment le système des personnages, la logique des actions et la temporalité » (Bertrand, 1985, p. 9). Pour Denis Bertrand, penser la question de l'espace « en dehors d'une conception globale et intégratrice de la signification discursive » (Bertrand, 1985, p. 17) est contradictoire et fallacieux. L'universitaire et critique littéraire Henri Mitterand stipule que l'espace a un rôle particulièrement intéressant dans la création littéraire. Il avance que «c'est le lieu qui fonde le récit, parce que l'évènement a besoin d'un ubi autant que d'un quid ou d'un quando » (Mitterand, 1986, p. 194). De là, à dire qu'il est primordial de penser la relation entre l'espace et les autres éléments du récit. Dans le même ordre d'idées, le critique littéraire Rolland Bourneuf propose d'approcher l'espace « dans ses rapports avec les personnages et les situations, avec le temps, avec l'action et le rythme du roman » (Bourneuf, 1970, p. 87). La théorie littéraire a longtemps été dominée par des études consacrées à la dimension temporelle. Toutefois, avant même le spatial turn, deux sémioticiens russes Mikhaïl Bakhtine et Youri Lotman ont revalorisé l'espace, en cela qu'ils ont démontré que les structures spatiales du récit sont primordiales à la production du sens (Ziethen, 2013, p. 4). Bakhtine propose pour approcher l'espace son concept de Chronotope pour mettre en lumière les constellations spatio-temporelles propres à une époque historique. Bakhtine définit son Chronotope comme «le principe générateur du sujet » ou encore " centres organisateurs des principaux évènements » (Ziethen, 2013, citant Bakhtine, 1978, p. 391). En ce sens, le temps est en fusion avec l'espace. Lotman met en lumière sa conception des relations spatiales, en avançant l'idée que les êtres humains sont profondément marqués par le visuel et le spatial. Dans ce sens, les êtres humains structurent l'espace sous l'effet de leur corps et de leur conscience corporelle. Et ce, selon des oppositions binaires. Dans ce sens, Lotman pense que ce " modèle spatial du monde devient dans [l]es textes un élément organisateur, autour duquel se construisent aussi ses caractéristiques non spatiales » (Ziethen, 2013, citant Lotman, 1973, p. 313). Ces théories sont d'importances pour approcher l'espace. Toutefois, notre étude recourt en grande partie à l'apport de Gaston Bachelard et plus particulièrement à sa conception de l'immensité intime.

\section{L'immensité intime}

La topoanalyse de Gaston Bachelard se défini comme « une étude psychologique systématique des sites de notre vie intime » (Bachelard, 1957, p. 27). Elle explicite les 
valeurs symboliques de paysages que le regard du narrateur ou d'autres personnages contemplent. Notre étude de l'espace du désert dans La nuit de feu serait amplement alimentée par l'immensité intime. Pour Bachelard, I'espace est essentiellement vécu par l'homme "dans les partialités de l'imagination » (Bachelard, 1957, p. 17). Autrement, la rêverie de l'immensité résulte de ce que le philosophe appelle un complexe spectaculaire où l'orgueil de voir est le noyau de la conscience de l'être contemplant. En somme, il s'agit : "d'une participation plus intime au mouvement de l'image » (Bachelard, 1957, p. 20). Nous aurons de ce fait à prouver dans la lecture du roman d'É. E. Schmitt que «l'impression de l'immensité est en nous » (Bachelard, 1957, p. 20).

C'est ainsi qu'en s'élançant dans l'immensité et en la contemplant que le rêveur paisible quitte l'« ici » pour s'élancer dans le lointain, bien au-delà de son environnement réel. On peut dire qu'il se projette dans un autre monde, dans un ailleurs. C'est donc une technique psychologique pour être ailleurs : « dans un ailleurs absolu qui fait barrage aux forces qui nous retiennent dans la prison de l'ici » (Bachelard, 1957, p. 233). L'espace vécu se voit ainsi marqué par l'ouverture vers un ailleurs naturel, immense, infini, sans bornes.

Bachelard considère que l'immensité intime est le produit d'une conscience de l'agrandissement (Bachelard, 1957, p. 169) en ce qu'elle mène vers une métamorphose de l'immensité dite extérieure en immensité intérieure. Par ricochet, le rêveur peut accéder à la beauté de l'immensité en y participant intimement en profondeur. Cela n'a de possibilité que parce que l'immensité loge déjà dans l'imagination de l'homme, au plus intime de lui-même. Il est nécessaire de réanimer sa flamme par le spectacle d'une immensité extérieur : « on peut entendre plus qu'un écho venu de l'extérieur, mais un appel intime de l'immensité » (Bachelard, 1957, p. 181). Si l'on pense autrement, on pourrait dire que si le rêveur permet à son antériorité de se déployer en contemplant l'espace immense, il va certainement éprouver « la consonance de l'immensité du monde et de la profondeur de l'être » (Bachelard, 1957, p. 173). Ce duo d'amour du rêveur et du monde comme le qualifie Bachelard lui-même fait du monde et, de l'homme deux créatures conjointes paradoxalement unies dans leur solitude.

Il est nécessaire selon Bachelard de bien sentir « la dilatation progressive de la rêverie Jusqu'au point suprême où l'immensité née intimement dans un sentiment d'extase dissout et absorbe, en quelque manière, le monde sensible» (1957, p. 220). Cette 
dilatation donne naissance à un effet d'apesanteur, voire d'ascension, ainsi qu'à une libération, de passibilité et d'unité qui résulte d'une ouverture de l'interne vers l'externe autrement de l'intimité de l'homme à l'intimité du monde. Cela nous conduit vers un fait qui est celui que l'immensité intime ne peut qu'être le produit de ce que Bachelard intitule une phénoménologie de l'expansion et de I'extase. Ce qui donc fait entrer l'infini en l'homme et lui permettant selon les mots de Bachelard de respirer cosmiquement (Bachelard, 1957, p. 180).

L'homme n'est selon Bachelard que le miroir de l'immensité. C'est à l'intérieur de I'homme que se déploie l'espace du dehors. Le regard est d'importance majeur parce qu'étant ce passage qui mène à la rêverie de l'immensité intime. On pourrait ainsi dire que l'œil contemple l'infini de l'espace et reflète le paysage contemplé. L'immensité se voit agrandie par la contemplation, par le regard. Il faut dire que c'est par la grandeur que l'espace intime et l'espace du monde deviennent consonnant jusqu'à ce qu'ils se trouvent dans un phénomène de fusion.

La rêverie est un peu particulière pour qui s'aventure dans le désert en ce que l'immensité vécu dans le désert par un rêveur retentit selon Bachelard en une intensité de l'être intime' (Bachelard, 1957, p. 185). Le philosophe se livre à une analyse du sentiment de l'immense vécu dans le désert, en étudiant Philippe Diolé pour qui « il faut vivre le désert tel qu'il se reflète à l'intérieur de l'errant! » (Diolé, cité par Bachelard, 1955, p. 178). Bachelard précise en citant Diolé que « ces montagnes en lambeaux, ces sables et ces fleuves morts, ces pierres et ce dur soleil », tout cet univers qui a le signe du désert est « annexé à l'espace du dedans » (Bachelard, 1957, p. 178). Le face à face du rêveur avec l'immensité du désert reste une expérience émouvante qui introduira le rêveur dans un espace psychiquement originelle parce que menant à la métamorphose de l'être. Il faut donc voire dans ce face à face entre le désert et l'être une « fusion entre l'être et cette espace concret » (Bachelard, 1957, p. 187). L'osmose qui survient entre I'homme et le désert devient un voyage qui conduira vers « l'ailleurs d'un autre monde » (Bachelard, 1957, p. 188).

\section{Les marques de l'immensité}

Le désert conçu par Schmitt semble infini. Ses frontières sont imprécises. Rachel bouvet dit dans ce sens : « [...] l'idée même de limite tend à disparaître lorsque l'œil est face à l'immensité du désert » (2006, p. 16). Le désert peut être saisi comme un 
véritable miroir de l'être contemplant l'infini, lieu de solitude du narrateur, l'espace de réflexions sur le néant, l'endroit de la révélation et de l'épiphanie. Rachel Bouvet considère que la confrontation de l'être avec l'immensité du désert mène vers :

La perte des repères, la mise en échec des signes, autrement dit un processus de désémiotisation, de même qu'un questionnement existentiel faisant affleurer des interrogations sur le néant, le caractère insaisissable de l'être, l'altérité, la mort. (Bouvet, 2006, p. 15-16)

On peut voir dans le désert schmittien l'espace d'une renaissance. C'est aussi l'espace d'un nouveau départ. Tout cela, se trouve vécu intimement par le narrateur. Dans l'intention d'éclairer cette intimité vécue, il nous parait opportun de procéder par prélèvements de plusieurs passages dans le roman.

Le premier cran à ouvrir pour franchir le texte Schmittien serait celui de la récurrence des marques de l'immensité telles qu'elles sont ressenties par les personnages. Il semble que ces marques émaillent à plusieurs égards toute la trame du roman, partout où il s'agit de personnages contemplant l'infini du désert. Cela se voit explicitement, non seulement dans le regard du narrateur mais aussi chez ses compagnons: «Tout devenait grandiose. Nous observions la terre à l'infini » (Schmitt, 2015, p. 97), « le regard braqué vers l'infini » (Schmitt, 2015, p. 73), « Je me dressai sur mes pieds et fixai les alentours en ressentant leur immense vacuité » (Schmitt, 2015, p. 84), « Je gisais, poussière au milieu de l'immense » (Schmitt, 2015, p. 89), "L'infini devant, derrière, sur les côtés » (Schmitt, 2015, p. 98). Cet accent mis sur l'immense nous est manifeste dans la répétition d'adjectif : « immense », « infini » « grandiose ». Il s'agit en somme d'adjectifs utilisés en vue d'une description du désert. Les mots sont des preuves variés, en saisir le sens, c'est bien comprendre les rêveries qui s'ouvrent, dans l'âme du narrateur, sous le signe des mots « infini » et « immense ». En effet, ces mots-là marquent de façon naturelle l'immensité intime parce qu'ayant des résonnances intimes.

Loin d'en tirer un résultat hâtif, il est bien clair que le narrateur É. E. Schmitt devant l'immensité du désert sent son cœur « se gorgeait tellement de sang » $(2015$, p. 100) de même qu'un sentiment de joie qu'on peut qualifier d'immense submergea le narrateur : «Une joie abyssale me submergea » (Schmitt, 2015, p. 98). On peut tirer de cela que la rêverie s'active dans la contemplation, ce qui signifie que la rêverie ne peut qu'être cet état amplement constitué dès l'instant initial. Bachelard considère que la rêverie 
laisse de côté l'objet proche pour se lancer dans l'ailleurs, le lointain. De ce fait, la rêverie de l'immense se forge dans la contemplation de l'infini.

Plus loin dans le récit Schmittien, le regard absorbe l'immense : « Je me dressai sur mes pieds et fixai les alentours en ressentant leur immense vacuité » (Schmitt, 2015, p. 84) le narrateur voit apparaitre un espace infini, celui du désert. Autour d'É.E. Schmitt, un vide sidérant. À mesure qu'il avance dans le désert, l'espace intérieur s'agrandit, s'étire, ce qui l'entraine dans un monde imaginaire, un monde où il va renaitre, un monde où il s'avance vers une expérience « inconnue ». Il semble attiré par la force de l'inconnu, de l'infini du désert: « je flairai que j'avais rendez-vous avec l'exceptionnel» (Schmitt, 2015, p. 8). La rêverie de l'immense se manifeste dans le ciel et les étoiles qui sont devenu « à portée de main » (Schmitt, 2015, p. 8). Il en résulte que « l'infini 'lui' souriait » (Schmitt, 2015, p. 8) en guise d'accueil dans la rêverie de l'immense. On pourra alors affirmer que l'espace extérieur s'agrandit, du vaste à l'immensité, de l'immensité à l'infinitude et à l'infinitude cosmique.

\section{L'immensité, une fusion corps-espace}

Dans le même ordre d'idées, le narrateur contemple l'infini du désert qui l'entoure et le réduisant au silence : «L'infini devant, derrière, sur les côtés... La rondeur du globe... Je ne pensais rien ; muet, je me réduisais à des yeux qui contemplaient » (Schmitt, 2015, p. 98). II est bien clair que la figure du désert dans le texte se construit également par rapport aux humains qui l'habitent ou le traversent. Ajoutons également qu'on retrouve plusieurs références au corps humain, à ses mouvements, à ses sensations. Le narrateur eut peur quand à l'idée de vivre tel un nomade dans le désert : « Un grelottement de peur me glaça et mes jambes flageolèrent » (Schmitt, 2015, p. 22). L'immensité du désert le trouble parce que sombre la nuit au point de l'introduire dans une terreur incontrôlable: «Mon cœur battit d'affolement » (Schmitt, 2015, p. 62). Le désert lui présente donc un danger, ce qui explique pourquoi il songe à penser la mort, pensée qui tourmente sa conscience et son cœur qui : « bat à se rompre » (Schmitt, 2015, p. 91). Sa respiration devient anormale : «Entendant sa respiration s'assourdir 》 (Schmitt, 2015, p. 86). Le narrateur et ses compagnons semblent dominer l'infinitude du désert : «Comme si la nature l'avait entendu, Un souffle nous enveloppa, assez hostile » (Schmitt, 2015, p. 39). Après les mouvements et les sensations, il nous parait important dans cette optique d'évoquer la posture du corps d'É. E. Schmitt, égaré dans le désert pour une nuit, il sera : « allongé sur le dos, en position de gisant, la figure face à l'étoile vespérale » (Schmitt, 
2015, p. 103) le corps immobile face au ciel. Cela donne à penser que cette posture n'est qu'un préambule à un mouvement qui va s'accomplir dans l'immobilité autrement, un mouvement tout à fait intérieur : «Je m'élève, je dépasse le sable, l'amas de rochers, et... je flotte » (Schmitt, 2015, p. 106). Ce mouvement est incontestablement imaginaire en ce qu'il décrit l'épiphanie vécue par le narrateur dans le désert. Il faut voir dans ce mouvement de l'être immobile une tentative de transcender le temps et l'espace.

Plusieurs faits attestent qu'une distorsion des dimensions se produit dans le texte. On peut citer l'infinitude qui semble charnellement vécut par le narrateur grâce à un phénomène d'expansion du corps. C'est d'ailleurs l'une des conclusions de Gaston Bachelard à savoir que : "L'espace poétique, puisqu'il est exprimé, prend des valeurs d'expansion » (Bachelard, 1957, p. 227). Cela semble évident dans ce passage : « Voilà que ça change déjà... J'ai l'impression que la force réintervient. Elle... elle m'agrandit ! Oui, elle distend mes membres, me rend colossal, m'étend aux dimensions du massif montagneux » (Schmitt, 2015, p. 106). Le narrateur est en plein rêverie de l'immense. Une force méconnue dilate ses membres, le rend immense. Cela traduit à bien des égards la conscience d'agrandissement théorisée par Bachelard dans la poétique de l'espace.

\section{L'immensité et le dédoublement du corps}

Ajoutons de ce fait que l'immense loge paisiblement dans la rêverie du narrateur. L'immensité du dehors stipule Bachelard se métamorphose en immensité intérieur. C'est bien grâces aux «contemplations des spectacles grandioses » ou encore à, l'âme qui médite et qui rêve tentant de trouver dans un objet le nid d'une immensité (Bachelard, 1957, p. 224) que l'immensité intime s'est opérée et que : « le spectacle extérieur vient aider à déplier une grandeur intime » (Bachelard, 1957, p. 218) Cependant, il n'y a de signes explicites montrant que les contours de cet espace intime sont clairement définis. Le corps du narrateur a connu une métamorphose, une dilatation de ses membres ou encore un dédoublement corporelle :

Incroyable : j'ai deux corps ! L'un sur terre, l'autre en l'air. Tandis que je distingue toujours, aussi ténu qu'un souvenir, le sable bloquant mes jambes et mon torse, je flotte Le prisonnier grelotte en bas et l'affranchi, léger, impalpable, s'élève tranquillement au-dessus du paysage, ne souffrant ni du froid ni du vent, délesté même de la respiration. (Schmitt, 2015, p. 106) 
Ici, deux corps sont mis en parallèle, qu'on peut saisir comme un détachement qui se produit fortuitement dans le silence total et la chaleur du sable : I'un affranchi, libre par une force qui le maintien en haut, et l'autre en bas prisonnier des immenses sables qui le bloquent. Si l'on pense autrement. II s'agit bel et bien de deux espaces l'un est intime et l'autre est extérieur. L'intime est l'affranchi par la force de l'immense, l'espace extérieur est celui du corps prisonnier, entouré des immenses dunes. Mais, ce n'est que lorsque le narrateur est devenu immobile, bloqué par la chaleur du sable que son véritable voyage intérieur commence. Le Philosophe Bachelard dit dans ce sens «l'immensité est le mouvement de l'homme immobile» (1957, p. 169). Pour s'épanouir, la rêverie de l'immense ne peut s'accomplir que dans le silence, la paisibilité et l'immobilisme corporelle.

Il importe de souligner que ce phénomène d'expansion se produit autrement dans La nuit de feu, c'est sous le mode d'une ascension décrite également dans la poétique de l'espace. En effet, Bachelard stipule que la dilatation à laquelle la rêverie a donné naissance, permet de se libérer de la pesanteur, ce qui donne à l'être le pouvoir « de respirer cosmiquement » (Bachelard, 1957, p. 180). Ajoutons que dans le dédoublement vécu par le narrateur, il se trouve « affranchi » « léger » pour ainsi flotter et s'élever « tranquillement au-dessus du paysage » (Schmitt, 2015, p. 106). Ce qui le mène à même à se " délester de la respiration » (Schmitt, 2015, p. 106) sur terre pour respirer cosmiquement. On peut dire également que la force qui a agrandit le narrateur lui a permis de connaitre une ascension.

Toujours sous le joug de l'expansion et de l'ascension, le narrateur prend une dimension cosmique : "Cette énergie inébranlable, indomptable, à l'œuvre dans L'univers, je m'absorbe en elle, J'en reçois des messages... » (Schmitt, 2015, p. 107) ou encore dans : « La force fonce. Je me laisse prendre. Elle me pénètre le corps, l'esprit. Me voici irradié ! J'épouse la lumière » (Schmitt, 2015, p. 107) Cela explique nettement la cosmicité de la rêverie chez le narrateur. Le narrateur s'absorbe dans l'énergie cosmique de l'univers formant un seul corps avec elle, un corps irradié, un corps fait de lumière : "Je ne formerai bientôt plus qu'un avec la force » (Schmitt, 2015, p. 107). Force est donc de constater que la rêverie de l'immense telle qu'elle se crée dans ce roman vient sous l'influence de ce que Bachelard intitule la phénoménologie de l'extension. Cela peut être saisi comme transformation de l'espace de l'extension en espace d'exaltation et de l'extase. On ne peut nier que la dialectique du haut et du bas, de la lumière et de l'ombre participe amplement dans cette exaltation ascensionnelle. 
Le dédoublement du narrateur en est un exemple précis, le corps qui se trouve en l'air est libre " affranchi », en une fusion, voire en osmose avec la force régissant l'univers. Il est: « irradiée », il forme corps avec la lumière, cette « énergie inébranlable, indomptable » (Schmitt, 2015, p. 107). Quant au corps « sur terre » à jambes bloquées par le sable, il est protégé du froid du désert, chaud dans le sarcophage de sable. De même qu'au terme de son expérience ascensionnelle qui lui a permis de fusionner avec la force du cosmos. Le narrateur sera « délicatement ramené sur terre » (Schmitt, 2015, p. 110) par la même force achevant donc « son voyage immobile » (Schmitt, 2015, p. 110).

Il se dégage de cela que chezÉ. E. Schmitt, la rêverie s'accomplit au travers d'une ascension fusionnelle du fait que le corps se dédouble devient aérien, il fusionne avec la nature et le cosmos. C'est ainsi que l'on peut affirmer, comme disait Bachelard, que l'expansion vécue par le personnage résulte du fait que : «l'immensité née intimement dans un sentiment d'extase dissout et absorbe, en quelque manière, le monde sensible » (1957, p. 177).

Dans La nuit de feu, le désert est intériorisé, l'immensité des sables et l'être s'interpénètrent. C'est bien une communication entre la conscience humaine et le cosmos. Cela corrobore le fait que l'homme dans le désert d'É. E. Schmitt : «se trouve dans un espace qui le contient et qui l'absorbe, mais qu'il contient et absorbe à son tour » (Real, cité par Vartian, 1989). On voit bien que le monde extérieur n'est plus séparé du monde intérieur du narrateur. Les frontières entre le corps et l'espace sont explicitement poreuses, permettant de ce fait, une osmose du paysage et de l'humain.

Ainsi, l'immensité intime peut être reliée au phénomène de fusion entre le corps et l'espace. Le corps et le cosmos entrent en fusion, cela nous est manifeste en ce que l'humain devient élémentaire. « Joie. Flamme. La force fonce. Je me laisse prendre. Elle me pénètre le Corps, l'esprit. Me voici irradié! J'épouse la lumière » (Schmitt, 2015, p. 107). Le narrateur se laisse irradier sans résistance, il se laisse pénétrer le corps, il devient lumière :

J'embrasse...

J'embrase...

Flamme.

Je suis flamme. Lumière croissante. Insoutenable. (Schmitt, 2015, p. 108)

La flamme franchit donc les limites du corps du narrateur, traverse sa peau et l'irradie au point de l'embraser, ce qui le rend : « éblouissant » et « fulgurant ». La force de 
l'immense enflamme son corps vivant et organique et lui permet de tout sentir : «Je sens tout. D'un coup, j'appréhende la totalité » (Schmitt, 2015, p. 108). Le narrateur entre en osmose avec la lumière. Le feu ardent du désert irradie son corps et le rend illimité :

Feu !

Soleil ardent. Je brûle, je fusionne, je perds mes limites,

J'entre dans le foyer.

Feu... (Schmitt, 2015, p. 109)

On note par ailleurs la présence du champ lexical se rapportant au feu et à la lumière, en référence à l'humain et au cosmos, en évoquant le mot « feu », «flamme », « lumière », « irradié », « éblouissant », « fulgurant », « incendié ».

\section{L'immensité intime ou l'ailleurs d'un autre monde}

Quand le narrateur se trouve « allongé sur le dos » dans le lit de sable « en position de gisant, la figure face à l'étoile vespérale » (Schmitt, 2015, p.103), il se lance dans la rêverie de l'immense atteignant un ailleurs extérieur et intime, spatiale et atemporel. En effet, l'expérience de l'immensité intime rend confuse non seulement les frontières entre le corps et l'espace, mais également elle permet de rendre poreuse les frontières entre l'espace et le temps. Cela donne naissance à un « ailleurs » au sens d'une zone entre le réel et l'imaginaire. L'espace infini que le narrateur voit et ressent en rêve semble ouvrir sur un ailleurs : « je pénètre dans l'au-delà du monde... » (Schmitt, 2015, p. 108). « Je ne me sentais plus isolé, étranger, mais fécondé, uni. La force qui tenait le Tout grouillait également en moi, j'incarnais l'un de ses maillons provisoires » (Schmitt, 2015, p. 127), cela va de soi avec la pensée bachelardienne à savoir que la contemplation de la grandeur du désert par le narrateur détermine une attitude si spéciale, un état d'âme impressionnant. La rêverie de ce fait met « le rêveur en dehors du monde prochain, devant un monde qui porte le signe d'un infini » (Bachelard, 1957, p. 180). II est clair que l'espace de l'ailleurs est bien celui qui se trouve dans la rêverie du narrateur, l'espace du rêve qui correspond à cet : « au-delà du monde » à ce « corridor » creusé par la force de l'immensité intime dans l'imagination du narrateur. De là, à dire que l'appel intime de l'immensité décrit par Bachelard introduit le narrateur dans un autre univers, situé au-delà des frontières spatiales. 
Par voie de conséquence, l'expérience vécue par le narrateur É. E. Schmitt dans le désert de Tamanrasset ne constitue pas seulement une aventure dans un espace ouvert, c'est bien dans « l'ailleurs d'un autre monde » (Bachelard, 1957, p. 188). Cela semble aller de soi avec l'expression Schmittienne : «Là, en moi, s'est creusé un corridor entre deux mondes » (Schmitt, 2015, p. 149).

\section{Conclusion}

Il ressort de l'analyse de ce roman philosophique que le désert infini et immense est un véritable miroir de l'être qui le contemple. É. E. Schmitt, comme homme et écrivain, a conçu le désert comme un espace de réflexions sur le néant, c'est aussi l'endroit de son épiphanie. On reconnait que l'immensité et l'infini du désert retentit en une immensité intérieur. Les marques de l'immense ont ouvert la voie vers une rêverie sous le signe de l'infini et de l'immense. Une rêverie qui s'est forgée par la contemplation du désert. C'est aussi la mobilité des personnages dans le désert qui fait que l'espace intérieur s'agrandit. Cela entraine tout contemplateur dans un monde imaginaire, un monde où il va renaitre où il s'avance vers I' « inconnue ». Ainsi, cela constitue un préambule à un mouvement qui se fait dans l'immobilité, mouvement tout à fait intérieur et imaginaire. Ce mouvement de l'être immobile décrit dans l'épisode du'sarcophage de sable'est compris comme une tentative de transcender l'espace et le temps. L'immensité intime se réalise aussi sous forme du phénomène d'expansion du corps, les membres du narrateur ont subi une dilatation. La conscience d'agrandissement permet aussi d'éclairer l'immensité intime chez le narrateur, c'est ainsi que l'immensité du dehors se métamorphose en immensité intérieur. Le narrateur connait également un dédoublement et un détachement qui se produit dans le silence total et la chaleur des immenses sables. Un corps bloqué par le sable et un autre en fusion avec la force qui régit le cosmos. Un corps en ascension permettant de respirer cosmiquement. De ce fait, La rêverie s'accomplit au travers d'une ascension fusionnelle. En se lançant dans la rêverie de l'immense le narrateur pénètre « dans l'au-delà du monde » (Schmitt, 2015, p. 108).

Évaluation : Évaluation anonyme par des pairs extérieurs.

Conflit d'intérêts : L'auteur n'a aucun conflit d'intérêts à déclarer.

Subvention : L'auteur n'a reçu aucun soutien financier pour ce travail.

Peer-review: Externally peer-reviewed.

Conflict of Interest: The author has no conflict of interest to declare.

Grant Support: The author declared that this study has received no financial support. 


\section{Bibliographie}

Alonso Aldama, J. (2009). « Espace et métalangage : défense du territoire ». Nouveaux actes sémiotiques (en ligne : http://revues.unilim.fr/nas/document.php?id=2893, consulté le 28 Aout 2020

Bachelard, G. (1957). La Poétique de l'espace. Paris : Presses universitaires de France.

Bakhtine, M. Daria, Olivier, (trad.) (1978). « formes du temps et du chronotope dans le roman », Esthétique et théorie du roman, Paris : Gallimard, p. 237-398.

Bertrand, D. (1985). L'espace et le sens : Germinal d'Émile Zola, Paris, Hadès.

Bourneuf, R. (1970). «L'Organisation de l'espace dans le roman ». Études littéraires, vol. 3, Num 1, Québec, 77-94. Bouvet, R. (2006). Pages du sable. Essai sur l'imaginaire du désert, Montréal (Québec) : XYZ éditeur.

Diolé, P. Le plus beau désert du monde, Paris. Albin Michel.

Greimas, A. J. (1976). «Pour une sémiotique topologique ». Sémiotique et sciences sociales. Paris : Éditions du Seuil. 129-157.

Heidegger, M. (1988). «L'Homme habite en poète » Essais et conférences. Paris : Gallimard, p. 224-245.

Levy, B. (1987) Géographie humaniste et littérature : l'espace existentiel dans la vie et l'oeuvre de Hermann Hesse (1877-1962). Thèse de doctorat : Univ. Genève, no. SES 326.

Lotman, Y. (1973). La Structure du texte artistique. Paris : Gallimard.

Merleau-Ponty, M. (2005). Phénoménologie de la perception. Paris : Gallimard.

Mitterand, H. (1980). « Le lieu et le sens: l'espace parisien dans Ferragus de Balzac », Le discours du roman, Paris : P.U.F. p. $189-212$.

Real, E. (1989). « Un espace pour le vide », Sud, N 85-86, p. 181-184.

Schmitt, É. E. (2015). La nuit de feu. Paris : Albin Michel.

Vartian, S. (2000). « Désert et immensité intime chez J.M.G. Le Clézo ». Dans Désert nomadisme, altérité. Cahier Figura. http://oic.uqam.ca/fr/articles/desert- et- immensite-intime chez-J-M-le-clezio. Consulté le 19 janvier 2020.

Warf, B. et S. Arias. (2009). «Introduction: The Reinsertion of Space into the Social Sciences and Humanities ». B. Warf et S. Arias (dirs.). The Spatial Turn: Interdisciplinary Perspectives. London : Routledge, P. 11- 35.

Ziethen, A. (2013). La littérature et l'espace. Arborescences, Num(3). https://doi.org/10.7202/1017363ar consulté le 23 juin 2020 12:02 